\title{
Does pregnancy affect medical ethical decision making?
}

Cathy Hammerman, Ofer Lavie, Eti Kornbluth, Jakob Rabinson, Michael S Schimmel and Arthur I Eidelman, Shaare Zedek Medical Center, Hebrew University - Hadassah Medical School, ferusalem, Israel

\begin{abstract}
Objective-We studied and compared the attitudes of pregnant women $v$ new mothers in an attempt to confirm changing patterns of maternal response towards medical ethical decision making in critically ill or malformed neonates.

Design-Data were obtained by questionnaires divided into three sections: 1. Sociodemographic; 2. Theoretical principles which might be utilised in the decision-making process; 3. Hypothetical case scenarios, each followed by possible treatment options. Results-Pregnant women ( $n=545)$ consistently requested less aggressive medical intervention for the hypothetical cases than did new mothers $(n=250)$ [Trisomy 18: 57\% v 42\%; $p=0.0004$; Asphyxia: $75 \%$ v 63\%; $p=0.0017$; Down's syndrome $81 \%$ v $62 \% ; p=0.0001 ; L B W 85 \%$ v $75 \%$; $p=0.004]$. Significant differences were also observed in the responses to the theoretical principles, with pregnant women attributing less importance to preserving life at all cost, while being more concerned with physical and emotional pain and suffering, with financial cost, and with the infant's potential for future productivity.
\end{abstract}

(Fournal of Medical Ethics 1998;24:409-413)

Keywords: Critically ill neonates; malformed neonates; medical ethics; expectant mothers; pregnancy; Down's syndrome

With increasingly sophisticated diagnostic technology, severe medical conditions which affect the fetus and neonate are more likely to be identified during pregnancy. As a result, ethical dilemmas concerning the medical management of such critically ill and/or malformed infants inevitably have to be faced much earlier, ie during pregnancy. Situations in which the best of medical care can be expected to result in the survival of a severely disabled child, or in which the chances of survival itself are minimal, necessitate ethically complex management decisions. However, pregnancy is described by clinicians and women alike as a time of emotional upheaval characterised by fluctuations in affective mood states. Increased maternal stress and anxiety can lead to injured self esteem, ${ }^{1}$ poor identification with motherhood, indifference towards, and even rejection of, the unborn fetus. ${ }^{2}$ These antepartum somatic and psychosocial stresses might affect pregnant women's medical ethical decision making concerning critically ill or malformed neonates.

This study was designed, therefore, to survey and compare the attitudes of pregnant women $\mathrm{v}$ postpartum mothers in the first year after delivery in attempt to document changing patterns of maternal response. The potential implications of such attitude shifts are that a woman, while pregnant, may make irrevocable decisions concerning the possible treatment or non-treatment of her fetus. These decisions can be expected to continue to impact upon the infant's condition during and after birth, via their influence on the aggressiveness of the response of medical staff to intrapartum fetal distress and on the utilisation of specialised support personnel during and after delivery. Furthermore, given the pregnant woman's evolving mood state and relationship to her fetus, this very same woman's desires as a new mother - when her previous treatment choices are still being implemented - may now be quite different. It is therefore incumbent upon the medical staff to anticipate and be sensitive to the impact that vicissitudes in mothers' emotional state may have on their - the mothers' - medical ethical decision making.

\section{Materials and methods}

A comprehensive questionnaire, devised from the joint input of a neonatologist, a social worker and an obstetrician, was used to study women's attitudes towards medical ethical decision making.

SUBJECTS

Cross-sectional studies were done on two subpopulations of women, taken from the same sociodemographic population base. 1. women during the 2 nd or 3rd trimester of pregnancy; and 
2. new mothers from six weeks to one year after birth. Women who were postpartum, but also pregnant (again) were excluded. The women were approached while waiting for routine visits to the clinics of the Shaare Zedek Medical Center, a university-affiliated teaching hospital. A volunteer spoke briefly with the woman, explaining the purpose of the study and assuring her of anonymity, before distributing the questionnaire, which the women filled out independently.

In order to minimise potential technical difficulties, questions called for one to two-word factual answers; none were open-ended. In recognition of the multiethnic diversity of our population, questionnaires were available in four different languages, and the women were able to choose that with which they felt most comfortable. The questionnaires were divided into three sections: 1.Sociodemographic data including age, level of religious observance and parity. Also included were questions about prior experience with infertility, prematurity and/or infants suffering from disabilities; 2 . Respondents were asked about their feelings about the pregnancy; about who should be involved in medical ethical decision making; and about the importance of certain principles which they might theoretically use to influence them in making medical ethical management decisions. These factors included preserving life at all costs, prognosis for future productivity and need to avoid physical pain or suffering; 3 . Respondents were then presented with five hypothetical case scenarios: Down's syndrome, extreme prematurity, severe perinatal asphyxia, Trisomy 18 and congenital heart disease. Case studies were described in great detail, in nontechnical language to prevent misunderstandings of either the factual aspects of the cases or the implications thereof. Each case was followed by three possible treatment options: maximal medical treatment (resuscitation and ventilatory assistance as needed), moderate medical treatment (up to, but not including resuscitation) or minimal medical treatment (feeding/warmth).

ANALYSIS OF DATA

Levels of educational attainment were grouped in ascending sequential order and then assigned a numerical score. Profession was ranked on a scale ranging from 1 which represented skilled professionals, to 6 which referred to unskilled workers. The scale is fully described elsewhere. ${ }^{3}$ Scores were compared using ANOVA. The theoretical principles were categorised as mildly, moderately or extremely important and were analysed as categorical variables. Responses to hypothetical case histories were categorised as the desire for maximal, moderate or minimal medical treatment and analysed as categorical variables. $\chi$ squaren tests were used to examine the hypothesis of independence between categorical variables of interestes in the different sub-population groups.

\section{Results}

STUDY SUBJECTS

Five hundred and forty-five pregnant women and 250 mothers within a year of delivery completedthe questionnaires. Comparative socio $\omega_{\sigma}$ demographic data are presented in table 1. New mothers were of higher parity than were pregnantw women $(2.9 \pm 2.4 \mathrm{v} 2.1 \pm 2.2 ; \mathrm{p}=0.001$; consistent with their having given birth to the one more childos that is still in utero in the pregnant group). Otherwise no significant differences were noted. Theo influence of parity on medical ethical decision? making was examined independently and shown not to affect treatment choices.

\section{PREVIOUS LIFE EXPERIENCES}

Previous life experiences which might potentially influence medical ethical decision making, inclư⿱⺈:ing prior infertility problems, having had a prex ous premature baby, and/or close association a brain damaged child were similarly distributês in the two groups (table 1). In addition these faco tors were analysed and not found to correlate witho treatment choice in either the pregnant women or the new mothers.

FEELINGS ABOUT PREGNANCY AND OBSTETRICIAN Significantly more women reported enjoying their pregnancy when interviewed during pregnancy aș compared with those interviewed after deliverys ( $87 \%$ v $78 \% ; p=0.005)$. Seventy seven per cent of pregnant women wanted their obstetrician to be involved in any communication regarding medica ethical decisions concerning their babies as compared with $53 \%$ of new mothers $(p=0.0015) \frac{I}{O}$

Table 1 Patient characteristics

\begin{tabular}{|c|c|c|c|}
\hline & $\begin{array}{l}\text { Pregnant } \\
(N=545)\end{array}$ & $\begin{array}{l}\text { New mothers } \\
(N=250)\end{array}$ & $p$ Value \\
\hline Age & $29.9 \pm 5.7$ & $29.0 \pm 5.8$ & 0.05 \\
\hline Parity & $2.1 \pm 2.2$ & $2.9 \pm 2.4$ & 0.001 \\
\hline Education & $2.8 \pm 1.0$ & $2.9 \pm 1.0$ & 0.19 \\
\hline Profession & $2.7 \pm 1.5$ & $2.8 \pm 1.3$ & 0.25 \\
\hline \multicolumn{4}{|l|}{ Infertility treatment } \\
\hline Medication & $8.7 \%$ & $6.9 \%$ & \\
\hline IVF & $3.7 \%$ & $2.9 \%$ & \\
\hline None & $87.6 \%$ & $90.2 \%$ & 0.565 \\
\hline \multirow{3}{*}{$\begin{array}{l}\text { Previous premature infant } \\
\text { Close association with brain } \\
\text { damaged child }\end{array}$} & $7.4 \%$ & $9.3 \%$ & 0.292 \\
\hline & & & \\
\hline & $9.8 \%$ & $6.5 \%$ & 0.185 \\
\hline
\end{tabular}


Table 2 Influence of religious observance on medical ethical decision making

\begin{tabular}{llll}
\hline & $\begin{array}{c}\text { Pregnant } \\
(N=545)\end{array}$ & $\begin{array}{l}\text { New mothers } \\
(N=250)\end{array}$ & p Value \\
\hline $\begin{array}{l}\text { \% of observant women } \\
\text { desiring max care }\end{array}$ & $71 \%$ & $85 \%$ & 0.0028 \\
$\begin{array}{l}\text { \% of non-observant women } \\
\text { desiring max care }\end{array}$ & $53 \%$ & $72 \%$ & 0.0057 \\
\hline
\end{tabular}

Table 3 Responses to hypothetical case scenarios: \% desiring maximal care

\begin{tabular}{llll}
\hline & $\begin{array}{l}\text { Pregnant } \\
(N=545)\end{array}$ & $\begin{array}{l}\text { New mothers } \\
(N=250)\end{array}$ & p Value \\
\hline CHD & $81 \%$ & $86 \%$ & 0.131 \\
LBW & $75 \%$ & $85 \%$ & 0.004 \\
Down's & $62 \%$ & $81 \%$ & 0.0001 \\
Asphyxia & $63 \%$ & $75 \%$ & 0.0017 \\
Trisomy 18 & $42 \%$ & $57 \%$ & 0.0004 \\
\hline
\end{tabular}

$\mathrm{CHD}=$ Congenital heart disease.

LBW $=$ Low birth weight .

\section{INFLUENCE OF RELIGION}

Each respondent graded her own level of religious practice as observant or non-observant. As there were differences in the distribution of level of religious observance in the postpartum population (67\% observant $v 33 \%$ non-observant), the choices for medical treatment in a representative case were analysed separately by level of religious observance and the responses of the pregnant women $\mathrm{v}$ the new mothers were compared within observant and non-observant groups separately (table 2). Down's syndrome was selected as the representative case scenario for this analysis because of its broad recognition within the community and intermediate severity, enabling a wide spectrum of responses. Within each subgroup, significantly more of the new mothers requested maximal medical treatment than did the pregnant women.

\section{DIVERSE CASE SCENARIOS}

Responses to the hypothetical case scenarios were also analysed and compared (table 3). Both groups were most aggressive in their desire to treat the case of congenital heart disease and least aggressive in the case of Trisomy 18, indicating an understanding of the relative severities of the various conditions. The new mothers consistently requested more aggressive medical intervention in each of the hypothetical cases, significant in all but the congenital heart disease scenario.

\section{PRINCIPLES}

Table 4 presents the responses to a series of general considerations which the respondents were asked to rate as to importance in influencing their medical ethical decision making. Significant
Table 4 Theoretical principles: \% considering extremely important in medical ethical decision making

\begin{tabular}{lllr}
\hline & $\begin{array}{c}\text { Pregnant } \\
(N=545)\end{array}$ & $\begin{array}{l}\text { New mothers } \\
(N=250)\end{array}$ & $p$ Value \\
\hline $\begin{array}{l}\text { Life at all cost } \\
\begin{array}{l}\text { Potential for future } \\
\text { productivity }\end{array}\end{array}$ & $64 \%$ & $77 \%$ & 0.0006 \\
$\begin{array}{l}\text { Infant's physical pain and } \\
\quad \text { suffering }\end{array}$ & $90 \%$ & $85 \%$ & 0.0237 \\
$\begin{array}{l}\text { Family's emotional suffering } \\
\text { Financial burden on family }\end{array}$ & $61 \%$ & $80 \%$ & 0.0525 \\
\hline
\end{tabular}

differences were observed in the responses of pregnant women $\mathrm{v}$ new mothers to each of the principles evaluated. New mothers felt it more important to preserve life at all cost, while pregnant women were more concerned with physical and emotional pain and suffering, with financial cost, and with the infant's potential for future productivity.

\section{Discussion}

In the highly technological atmosphere of modern intensive care medicine, we must periodically step back and reassess the balance between the commitment to preserve human life and the medical philosophical dictate to do no harm. Because fetuses and neonates have no autonomous decision-making capacity and cannot yet express their desires, they present a unique ethical challenge. " "Society generally presumes that parents should exercise the right to refuse medical treatment when nonautonomous children cannot do so for themselves"(AAP guidelines). ${ }^{5}$ Clearly, it is the family's prerogative, as the usual best advocates of the infant, to be intimately involved in such decision making. However, at the same time it is incumbent upon us to realise that no one who is intimately involved can be totally objective or completely free of any conflict of interest. ${ }^{6}$ Furthermore, parents in such situations are emotionally vulnerable, generally unprepared and usually still reeling from the shock of not having the normal, healthy baby they had heretofore envisioned. They must struggle with violently conflicting emotions. The love, concern and hope for the baby's wellbeing is counterbalanced by the grief, disappointment and feelings of guilt over the reality of their imperfect infant. They may be afraid of losing the baby while, at the same time, be terrified of the impact his/her possible survival will have on their wellbeing as a family. ${ }^{7}$ Decisions concerning the limitation of medical treatment are never simple, however there are those who feel that it is somewhat easier to forgo life-sustaining treatment in a fetus or neonate because a deep psychological bond between the mother and infant has not yet been established. 
We have shown that pregnant women consistently requested less aggressive medical treatment than did new mothers when making medical management decisions regarding critically ill or malformed infants. Several possible explanations for this come to mind, however, all are only speculative. Possibly, as alluded to above, the mother has not yet bonded strongly to her infant and thus while pregnant, the fetus remains an "abstract". However, once the baby is born, because of the strengthened emotional bond developed immediately, the mother begins to personalise the questions and becomes more aggressive in her treatment desires.

Conversely, the exact opposite may be true. Pregnancy is known to be fraught with prognostic uncertainty and pregnant women tend to be preoccupied with concerns and fears for the physical wellbeing of the unknown child within. Given this state of mind, the hypothetical cases presented in the questionnaire might be analysed in a very personal fashion, ie this could well be my baby - can $I$ cope? What would $I$ do if it happened to me? Since in her present condition, the already stressed pregnant woman may not feel capable of handling such a devastating problem, she may be more likely to request less aggressive medical intervention. Once her child is born, there is a relief derived from the knowledge that her baby is no longer an unknown. Most likely the baby was born healthy, and the hypothetical cases are now analysed in a more abstract and impersonal fashion. These women are more apt to make decisions based on a sense of what they perceive to be societally and theoretically correct rather than personally comfortable. Furthermore, even if her baby has not been born completely healthy, anticipated problems are generally worse than the reality. Pinelli ${ }^{8}$ demonstrated that mothers of impaired infants often expressed great anxiety in anticipation of caring for their infants at home, however they generally became more comfortable with their infants after actively assuming responsibility for their care.

\section{Emotional disequilibrium}

In addition, pregnancy itself is often accompanied by emotional disequilibrium, both as a result of pregnancy-induced endocrinological alterations contributing to emotional stress and of preoccupation with physical changes causing a disruption in body image. ${ }^{9}$ Excessive stress during pregnancy can precipitate depressive symptoms ${ }^{8}$ which, in turn, can lead to poor self esteem, poor identification with motherhood, indifference towards, and even rejection of, the unborn fetus. ${ }^{3}$ To the extent that the pregnant woman is focused predomi- nantly upon herself, thoughts about carrying an ill or malformed infant are likely to be viewed from $\bar{\varnothing}$. the perspective of this fetus's impact upon her life. This is not to imply that the pregnant woman is not interested in the child's welfare, but rather that the vantage point from which she perceives what is $\frac{\bar{\sigma}}{\bar{N}}$ best for this child may be somewhat shifted.

\section{Theoretical principles}

These sorts of reasoning patterns can also be helpful in understanding the responses to the $\vec{\rho}$ importance of various theoretical principles in decision making. Pregnant women were more concerned with physical and emotional pain and suffering and with financial cost, which is consist-o ent with a preoccupation with pregnancy- ${ }^{\circ}$ associated physical and emotional changes and discomforts which they themselves are undergoing. Meanwhile, new mothers felt it more impor- $-\vec{c}$ tant to preserve life at all cost, again hypothetically consistent with their ability, at this point in the $\frac{\mathscr{\Phi}}{3}$ process, to focus on the infant as an independent being with separate needs. It is important to emphasise that any thought processes which we have potentially attributed to the study popule tions are merely speculative. None of this can directly derived from our data.

Regardless of the rationale, the importance of these observations relates to the fact that medical management decisions made by the pregnant $\stackrel{\mathbb{Q}}{\Omega}$ mother, although generally not irrevocable, may $\overrightarrow{\overrightarrow{0}}$ nevertheless have a crucial impact on continuing 3 care, regarding the care of her infant both during and after delivery, at which time her perspective may have changed. If, for instance, she has elected not to deliver in a tertiary centre where extensive support services are readily available and she subsequently does desire to avail herself of such specialised personnel and services, she may be forced to be separated from her infant early after delivery 응 when maternal-infant bonding is critical; if she has elected not to undergo a caesarean for fetal $\frac{D}{0}$ distress and the infant survives but sustains $\frac{\bar{N}}{N}$ further intrapartum damage, she may suffer deep o regrets; and finally, if she has elected to terminate $N$ the pregnancy, she may now suffer from confusion $N_{\omega}$ and feelings of guilt. Awareness of trends in maternal decision making such as those demon-co strated here can enable us to understand better $\frac{}{\Phi}$ mothers' desires in the context of their current $\stackrel{\oplus}{+}$ health status and to facilitate more effectively our $\underline{0}$ role as intermediaries in the decision-making $\stackrel{\vec{Q}}{\vec{Q}}$

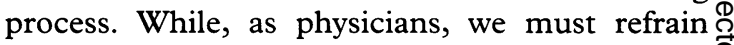
from becoming paternalistic, we do retain an obli- $\frac{\mathbb{Q}}{2}$ gation to exercise some moral judgment rather than blindly following patient demands. ${ }^{10}$ 
Although questionnaire-based studies may not accurately portray the emotional turmoil of an acute clinical crisis situation, they do provide a reasonable simulation and perhaps allow for more carefully considered responses without the overlay of emotional stress conferred by having a seriously ill baby. ${ }^{112}{ }^{13}$ We elected not to administer the questionnaire sequentially to the same group of women before and after delivery for two reasons. Firstly, it was of utmost importance, considering the imperative to maintain privacy in our population and the sensitivity of the issues in question, that we guarantee the women anonymity in order to assure maximal compliance. Secondly, in an attempt to prevent recall of previous answers to the same questions (administered within a period of several months) from biasing the responses of the new mothers, an independent group of mothers was studied.

Finally, this study was NOT aimed at providing specific numbers. Clearly, the precise percentages will vary from population to population. Rather, our aim was to define patterns of response during pregnancy as opposed to postpartum. Thus, although our study population may be ethnically distinctive, we do not feel that this cultural uniqueness affects our conclusions. Both study groups were sampled from the same overall population and were sociodemographically similar to each other. As such, ethnocultural parameters were balanced in both study groups. Furthermore, the patterns of the responses between the pregnant women and the new mothers were similar at every level of religious observance. We therefore believe that the different responses observed do not reflect the cultural/religious background of our population, but rather represent the differences in their pregnancy status.

In summary, pregnant women in our population consistently chose less aggressive treatment than did new mothers in response to questions concerning medical management of critically ill or malformed neonates. The implications of such shifts in attitude can be overwhelming. A woman, while pregnant, may make decisions concerning the treatment or non-treatment of her fetus which will continue to impact upon his/her care during and after birth. We speculate that the differences in responses observed reflect the effects of the somatic and psychosocial stresses of pregnancy on ethical decision making, coupled with the new mother's propensity to relate to the questions in an abstract rather than a personal construct once her baby is born and known to be healthy. However, alternative explanations are possible. Future studies might focus on the attitudes of fathers, and of mothers of seriously ill newborns, to similar issues in order to gain further insight.

\section{Acknowledgement}

Supported in part by the Joyce and Sidney Gamburg Neonatal Research Fund and by the generous support of $\mathrm{Mr}$ and Mrs Stephen Hammerman.

Cathy Hammerman, MD, Michael Schimmel, MD and Arthur I Eidelman, MD, are in the Department of Neonatology, Shaare Zedek Medical Center, Hebrew University-Hadassah Medical school, ferusalem, Isreal. Ofer Lavie, $M D$, and Fakob Rabinson, $M D$, are in the Department of Obstetrics/Gynecology and Eti Kornbluth, $M A$, is in the Department of Social Work at the same institution.

\section{References}

1 Colletta ND. At risk for depression: a study of young mothers. Fournal of Genetic Psychology 1983; 142:301-10.

2 Koniak-Griffin D, Walker DS, de Traversay J. Predictors of depression symptoms in pregnant adolescents: fournal of Perinatology 1996; 16:69-76.

3 Harlap S, Davies AM, Grover NB, Prywes R. The Jerusalem perinatal study: the first decade 1964-1973. Israel fournal of Medical Science 1977; 13:1073-9.

4 Steinberg A. Meningomyelocele in the neonate: medical and ethical considerations. Fournal of Perinatology 1991; 11:51-6.

5 Committee on Bioethics. Guidelines on forgoing life sustaining medical treatment. Pediatrics 1994 ; 93: 532-6.

6 Peabody JL, Martin GI. From how small is too small to how much is too much: ethical issues at the limits of neonatal viability. Clinics in Perinatology 1996; 23:473-89.

7 Rostain AL, Bhutani VK. Ethical dilemmas of neonatalperinatal surgery. Clinics in Perinatology 1989; 16:275-302.

8 Pinelli JM. A comparison of mothers' concerns regarding the care-taking tasks of newborns with congenital heart disease before and after assuming their care. Fournal of Advanced Nursing $1981 ; 6: 261-70$.

9 Alfonso DD, Mayberry LJ. Common stressors reported by a group of childbearing American women. Health Care Women International 1990; 11:331-45.

10 Paris JJ, Schreiber MD. Physicians' refusal to provide life-prolonging medical interventions. Clinics in Perinatology 1996; 233:563-71.

11 Byrne P, Tyebkhan J, Laing LM. Ethical decision making and neonatal resuscitation. Seminars in Perinatology 1994; 18:36-41.

12 Segal S. Ethical issues presented by children with congenital anomalies. Seminars in Perinatology 1992; 16:369-72.

13 Todres ID, Guillemin J, Grodin M, Batten D. Life-saving therapy for newborns: a questionnaire survey in the state of Massachussetts. Pediatrics 1988; 81:643-9. 